\title{
Narcolepsy-cataplexy and loss of sphincter control
}

\author{
AN Vgontzas, SE Sollenberger, A Kales, EO Bixler, A Vela-Bueno
}

\begin{abstract}
Summary
We describe the case of a 34-year-old man who presented intermittent faecal incontinence as a manifestation of cataplexy. The patient's sleep history was positive for the full narcoleptic tetrad (sleep attacks, cataplexy, sleep paralysis and hypnagogic hallucinations) while extensive neuropsychiatric work up was negative for any neurologic or psychiatric illness. Repeat polysomnograms (including a polysomnogram with a full seizure montage) were positive for pathologic sleepiness, but there was no evidence of a seizure disorder. The course of the patient's symptomatology and the favourable response of his symptoms to stimulants and imipramine support the theory that his intermittent loss of sphincter control is part of his narcolepsy-cataplexy.
\end{abstract}

Keywords: narcolepsy-cataplexy, sphincter control

Narcolepsy is a disorder of excessive sleepiness characterised by daytime sleep attacks, cataplexy, sleep paralysis and hypnagogic hallucinations. ${ }^{1-3}$ Cataplexy occurs in $60-70 \%$ of all cases of narcolepsy and is considered a pathognomonic symptom of the disorder. Clinical features of the cataplectic attack may include various degrees of weakness of the entire voluntary musculature or more frequently certain muscle groups as manifested by buckling of the knees, 'clumsiness' or head droop and sagging of the jaw. Less frequently, a cataplectic attack involves speech, respiration and extraocular muscles. The sphincter muscles are considered to be uninvolved in a cataplectic attack ${ }^{1}$; loss of sphincter control as a feature of cataplexy is not mentioned in textbooks on sleep disorders medicine. ${ }^{2}$

Sleep Research and Treatment Center, Department of Psychiatry, Pennsylvania State University College of Medicine, Hershey, PA 17033, USA

AN Vgontzas

A Kales

EO Bixler

A Vela-Bueno

\section{Chambersburg}

Hospital, Chambers-

burg, PA, USA

SE Sollenberger

Accepted 22 November 1995

\section{Case report}

A 34-year-old married man was referred to our Sleep Disorders Clinic for evaluation of symptoms consistent with narcolepsy. Approximately six months earlier the patient had an eight-day period in which he experienced fairly persistent diplopia and weakness of his left arm and left leg with intermittent episodes of faecal incontinence not associated with urgency. The episode resolved without any residual symptoms. A clinical examination by his family physician and several laboratory tests including electroencephalogram (EEG) and computed tomography scan of the brain were negative for abnormal findings. However, because the patient continued to experience blurred vision, left-sided weakness and faecal incontinence on an intermittent basis, but not concurrently, and the patient was unsure whether these symptoms might be associated with any emotional condition, he was referred to a neurologist (SES) for a thorough evaluation.

His initial neuroexamination was positive for right-beating nystagmus in right gaze and a slight increase in deep tendon reflexes in the left upper extremity as compared to the right. There was no sensory level in the trunk, and no 'saddle' anesthesia was demonstrated. The remainder of the neuroexamination was normal. A cerebrospinal fluid examination including cultures for acid-fast bacilli and cryptococcus and serologic tests for Lyme disease and syphilis, magnetic resonance imaging of the brain, brainstem, and cervical spine, and nerve conduction studies (both motor and sensory) in the lower extremities and left upper extremity were normal. Because of an additional history of excessive daytime sleepiness, the patient was referred to our sleep disorders clinic for further evaluation.

A thorough sleep history revealed a 10- to 15-year history of excessive sleepiness and sleep attacks which occurred in the classroom, at work and while driving (four years ago he had wrecked his car). During the last four years, the patient's sleepiness had become worse; he complained of brief periods of behavioural automatism ('memory lapses') and presented brief episodes of loss of muscle control such as buckling of his knees, dropping of tools from his hands and head drops to the front which sometimes were associated with laughter. Also, more recently, he began experiencing vivid hypnagogic auditory hallucinations and episodes of sleep paralysis. His family history was significant for his mother and a maternal aunt and uncle with histories of excessive daytime sleepiness. A comprehensive psychiatric evaluation including a thorough psychiatric history, mental status examination and psychological testing (Minnesota Multiphasic Personality Inventory) was negative for a psychiatric disorder.

The patient was evaluated in the Sleep Laboratory for one eight-hour night recording and two one-hour daytime naps according to techniques previously described. ${ }^{4-6}$ All records were scored for sleep according to standard criteria. ${ }^{4}$ His sleep laboratory testing was negative for obstructive or central sleep apnea and nocturnal myoclonus. No sleep-onset rapid-eye movement (REM) periods were noted. However, he demonstrated shortened sleep latencies during the two naps (6 and 10 


\section{Clinical features and management of narcolepsy}

- sleep attacks are brief, irresistible, episodes of sleep

- sleep attacks are sometimes associated with dreaming

- duration of sleep attacks varies from a few to thirty minutes

- most patients feel refreshed after a sleep attack

- chronic excessive daytime sleepiness between attacks of sleep

- methylphenidate and amphetamines are the primary and most effective treatment

- pemoline and modafinil have been reported to have moderate and substantial effects, respectively

- therapeutic naps may decrease the need for medication

\section{Clinical features and management of cataplexy}

- attacks of sudden reduction or loss of muscle tone

- attacks are most often triggered by emotional stimuli, ie, surprise, anger, laughter

- may be accompanied by hypnagogic hallucinations

- consciousness is almost always maintained

- last from a few seconds to several minutes

- tricyclics (imipramine, clomipramine) in low doses $(10-50 \mathrm{mg}$ ) are the treatment of choice

- fluoxetine and gamma-hydroxybutyrate have been reported to have significant anticataplectic effects

\section{Learning/summary points}

- loss of sphincter control may be a rare manifestation of cataplexy

- cataplexy should be included in the differential list of neurologic disorders associated with loss of sphincter control

respectively. Evaluation of the sleep EEG and $\stackrel{\mathbb{D}}{\circ}$ the videotape was negative for paroxysmal $\stackrel{C}{.}$ events or abnormal movements. Following this $\overrightarrow{\overrightarrow{\mathrm{F}}}$ sleep study, the patient was prescribed imipra $-\overrightarrow{0}$ mine $10 \mathrm{mg}$ each morning. He has been free of $\frac{\bar{O}}{0}$ episodes of faecal incontinence for the last nine $\frac{\overline{\bar{p}}}{\overline{\frac{p}{\sigma}}}$ months.

\section{Discussion}

Our case demonstrates that loss of sphincter $\overrightarrow{\vec{\omega}}$ control may be, though rarely, a cataplectic manifestation. This is supported by the presence of the narcoleptic tetrad (daytime sleep? attacks, cataplexy, sleep paralysis and hypna- N gogic hallucinations); positive family history of of excessive sleepiness; objective documentation. of pathologic sleepiness in the sleep laboratory and lack of evidence of seizure activity; a $\circ$ significant response of his faecal incontinence to treatment with methylphenidate and a more $>$ definite response to imipramine; and lack of evidence of any disease of the central or peripheral nervous system or psychiatric dis $-\vec{\theta}$ order (during initial and repeat follow-up visits), which might have explained the patient's symptomatology. The presence of ob-o jective neurological findings (in our case transient), particularly oculomotor disorders, is not infrequent in narcoleptics, especially $\frac{\infty}{\mathbb{D}}$ when the condition is familial. ${ }^{1}$

Thiele and Bernhardt in 1933 described a $\overline{\bar{O}}$ patient with narcolepsy-cataplexy and episodes of enuresis. ${ }^{7}$ However, they attributed the enuretic episodes to seizure activity rather than to cataplexy. In our case, sleep studies, a fully 3 preserved consciousness during the episodes, $\dot{0}$ and a positive response to methylphenidate and imipramine, do not support such a $\delta$ possibility. A cataplectic attack can affect any voluntary muscle including ocular muscles and 음 diaphragm, and there is no theoretical reason $I$ that sphincter muscles should be spared.

In summary, loss of sphincter control may occur as a feature, though very rarely, of cataplexy. Also, cataplexy should be included $N$ in the differential list of neurologic disorders $\omega$ which may result in loss of sphincter control, and an assessment for the presence of narcolepsy-cataplexy in atypical cases may provide $\stackrel{\oplus}{\oplus}$ useful diagnostic clues.

During the second nap, his sleep and REM latencies were six mi:cutes and 37 minutes,

1 Roth B. Narcolepsy and hypersomnia. Basel: S Karger, 1980. 2 Guilleminault C. Narcolepsy syndrome. In: Kryger MH, Roth T, Dement WC, eds. Principles and practice of sleep medicine. Philadelphia: WB Saunders Company, 1994; pp 549-61.

3 Kales A, Cadieux RJ, Soldatos CR, et al. Narcolepsycataplexy. I. Clinical and electrophysiologic characteristics. Arch Neurol 1982; 39: 164-8.

4 Rechtschaffen A, Kales A. A manual of standardized terminology, techniques and scoring system for the sleep stages terminology, techniques and scoring system for the sleep stages
of human subjects. Washington, DC: Public Health Service, of human subjects. Washington, DC: Publice,
US Government Printing Office, 1968.
5 Kales A, Bixler EO, Soldatos RJ, Cadieux RJ, Manfredi R, Vela-Bueno A. Narcolepsy/cataplexy. IV. Diagnostic value of daytime nap recordings. Acta Neurol Scand 1987; 75: 223-30.

6 Roth B, Nevsimalova, Sonka K, Docekal P. A quantitative 8 polygraphic study of daytime somnolence and sleep in patients with excessive diurnal sleepiness. Schweiz Arch Neurol Psychiatr 1984; 135: 265-72.

7 Thiele R, Bernhardt H. Beiträge zur Kenntniss der Narkolepsie. Berlin: S Karger, 1933. 